\title{
On asymptotic stability of solitary waves
}

\author{
Robert L. Pego \\ Department of Mathematics, University of Maryland, College Park, MD 20742, USA
}

and

Michael I. Weinstein

Department of Mathematics, University of Michigan, Ann Arbor, MI 48109, USA

Received 27 November 1991; accepted for publication 9 December 1991

Communicated by D.D. Holm

\begin{abstract}
Solitary waves of the Korteweg-de Vries equation (KdV) and of the generalized Korteweg-de Vries equation, gKdV, with "generic" nonlinearity, are orbitally asymptotically stable. A solution, beginning in a neighborhood of the solitary wave, has the form of a solitary wave with time varying speed and center of mass (respectively, $c(t)$ and $\gamma(t)$ ) plus an error term, $v(, t)$, which tends to zero exponentially fast as $t \rightarrow+\infty$, in a suitably weighted function space. The solution is decomposed into a bound state part, governed by the modulating parameters $c(t)$ and $\gamma(t)$, sometimes called collective coordinates, and an infinite dimensional dispersive part, which in the appropriate space is shown to behave dissipatively.
\end{abstract}

\section{Introduction}

Solitary waves are a class of finite energy localized solutions of nonlinear dispersive equations of Hamiltonian type. In many systems they are observed to participate in a dominant way in the dynamics of general solutions $\#$.

With a view toward a mathematical understanding of this, it is natural to consider the problem of solitary wave stability. An extensive literature has developed on this question, beginning with the pioneering work of Benjamin [3] on the stability of the soliton of the Korteweg-de Vries equation [4], an equation first studied in the context of long wave propagation in shallow water. Here, the notion of stability used is "orbital stability", which has its direct counterpart for finite dimensional systems [5].

* This research was supported in part by grants from the National Science Foundation.

\#1 This is precisely the case in completely integrable equations, such of $\mathrm{KdV}$, which are solvable using the inverse scattering transform (IST). Here the solitary waves are called solitons. (See for example refs. $[1,2]$.)
By the orbit of the solitary wave, $u^{\text {sol }}$, we mean the set $O\left(u^{\text {sol }}\right)=\left\{g u^{\text {sol. }}: g \in \mathfrak{G}\right\}$, where $(\mathfrak{5}$ denotes the group of symmetries of the equation. Roughly speaking, the solitary wave is orbitally stable if a small deviation of $g u^{\text {sol }}$ from the initial data, for some $g \in \mathfrak{G}$, implies that for every $t>0$, there is a $g(t) \in(5)$, such that the deviation of the solution from $g(t) u^{\text {sol }}$ is small. Here the deviation is measured in a suitable norm, which is dictated by the conserved integrals of the system.

This notion of stability, while capturing some details of the dynamics, does not address the question of what the asymptotic state of the system is. A priori it is possible that the solution "wanders" close to the solitary wave orbit without settling down to some well defined asymptotic state. The approach used to obtain these stability results cannot capture precise asymptotic behavior, as norms constructed from conserved integrals are necessarily insensitive to dispersive phenomena; if solutions are asymptotically attracted to some asymptotic state, the mechanism will be, in part, the dispersive radiation of excess energy.

A more refined approach to the stability question 
is to work with norms which are sensitive to dispersive phenomena. In such norms, one seeks to prove "orbital asymptotic stability". In the setting of this paper this notion of stability takes the following form: the solitary wave is orbitally asymptotically stable if a small deviation of $g u^{\text {sol }}$ from the initial data, for some $g \in \mathfrak{G}$, implies that there exists a function $g(t)$, with values in 6 , such that $g(t) \rightarrow g_{\infty}$, and the solution converges to $g_{\infty} u^{\text {sol }}$ in norm as $t \rightarrow \infty$. (In finite dimensional conservative systems this approach has no analogue; (i) the source of dispersion is the continuous spectrum, which is absent in finite dimensional systems, and (ii) all norms are equivalent in finite dimensional spaces.) This program has been carried out for a class of nonlinear Schrödinger systems by Soffer and Weinstein in refs. [6-8].

In this paper we consider the asymptotic stability of solitary waves of the Korteweg-de Vries equation

$$
\partial_{t} u+\partial_{x}\left(\frac{1}{2} u^{2}+\partial_{x}^{2} u\right)=0 \text {. }
$$

Since our methods do not depend on the property of complete integrability of $\mathrm{KdV}$ [2], we can obtain results for the generalized Korteweg-de Vries equation (gKdV)

$\partial_{t} u+\partial_{x}\left[f(u)+\partial_{x}^{2} u\right]=0$.

For simplicity, we work with the particular nonlinearity $f(u)=u^{p+1} /(p+1)$. Our methods apply to give results for more general nonlinearities. Detailed proofs of the results in this paper and further discussion will appear in ref. [9].

Eq. (1.1) has solitary traveling wave solutions of the form: $u(x, t)=u_{c}(x-c t+\gamma), \gamma \in \mathbb{R}^{1}, c>0$, where the solitary wave profile $u_{c}(\xi)$ is the decaying solution of the equation

$$
-\partial_{\xi}^{2} u_{c}+c u_{c}-f\left(u_{c}\right)=0 \text {. }
$$

Solitary waves of any speed $c>0$ will exist, for example, under the hypotheses that $f$ is $\mathrm{C}^{1}$ and convex for $u>0$ with $f(0)=0=f^{\prime}(0)$ and $f(u) / u \rightarrow \infty$ as $u$ increases. For the case of a power nonlinearity above, we have $u_{c}(\xi)=c^{1 / p} u_{1}\left(c^{1 / 2} \xi\right)$. In this case, $\mathrm{O}\left(u^{\text {sol }}\right)=\left\{c^{1 / p} u_{1}\left(c^{1 / 2}(\cdot+\gamma)\right): c>0, \gamma \in \mathbb{R}^{1}\right\}$.

We have the following result. Below, for $\sigma>0$, $\mathrm{H}_{\sigma}^{1}$ denotes the space of functions $f$ such that $\mathrm{e}^{\sigma x} f(x)$ lies in $H^{1}\left(\mathbb{R}^{1}\right)$, with norm given by
$\|f\|_{\mathbf{H}_{\sigma}^{\prime}}^{2}=\int_{\mathbb{R}^{1}}\left[|f(x)|^{2}+\left|\partial_{x} f(x)\right|^{2}\right] \mathrm{e}^{2 \sigma x} \mathrm{~d} x$.

Theorem 1. Let $p=1$ or $1<p<4$ (except possibly for a finite set of values of $p$; see proposition 3 ). Let $c_{0}>0$ and let $u_{c 0}$ be the corresponding solitary wave profile. Let $0<\sigma<\sqrt{c_{0}}$ be a positive number, which is suitably chosen. Consider the initial value problem for (1.1) with data, $u(x, 0)=u_{c 0}(x)+v_{0}(x)$. Assume that $u(\cdot, 0) \in \mathrm{H}^{2}$ and that the perturbation, $v_{0}$, of the solitary wave is sufficiently small in the $\mathrm{H}^{1} \cap \mathrm{H}_{\sigma}^{1}$ norm. Then, the solution of $\mathrm{gKdV}$ has the form of a "nonlinear bound state" ( solitary traveling wave) plus a part which disperses to zero. Precisely, there exist functions $c(t)$ and $\gamma(t)$ such that $c^{+}=\lim _{t \rightarrow+\infty} c(t), \gamma^{+}=\lim _{t \rightarrow+\infty} \gamma(t)$ exist, and such that

$u(x, t)=u_{c(t)}(x-\theta(t))+v(x-\theta(t), t)$,

where $\theta(t)=\gamma(t)+\int_{0}^{t} c(s) \mathrm{d} s$, and

$\|v(\cdot, t)\|_{\mathbf{H}_{\sigma}^{1} \rightarrow 0,} \quad c(t) \rightarrow c^{+}, \quad \gamma(t) \rightarrow \gamma^{+}$

at an exponential rate as $t \rightarrow+\infty$.

The $\mathrm{H}^{1}$ orbital stability of the solitary wave or soliton of $\mathrm{KdV}(p=1)$, was proved in ref. [3] (see also ref. [10]). Work that followed (see for example refs. [9,11-15]) led to the result that the solitary wave $u_{c 0}$ is orbitally stable, if and only if, the "momentum" $\mathscr{N}\left[u_{c}\right] \equiv \frac{1}{2} \int u_{c}^{2}$ is a strictly increasing function of $c$ at $c_{0}$. (This reduces to the range $1<p<4$ for the case of the power nonlinearity $f(u)=u^{p+1} /(p+1)$.) Here, orbital stability means precisely the following: given any $\epsilon>0$, there is a $\delta(\epsilon)>0$, such that if

$\inf _{\gamma \in \mathbb{R}^{1}}\left\|u_{c}(\cdot+\gamma)-u_{0}(\cdot)\right\|_{\mathrm{H}^{1}}<\delta$,

then for all $t$,

$\inf _{\gamma \in \mathbb{R}^{1}}\left\|u_{c}(\cdot+\gamma)-u(\cdot, t)\right\|_{\mathrm{H}^{1}}<\epsilon$.

Thus if at time $t=0$ the solution's "shape" is $\mathrm{H}^{1}$-close to that of some spatial translate of the solitary wave, then for any $t>0$ the solution is $\mathrm{H}^{1}$-close to some $t$ dependent translate of the solitary wave. The $\mathrm{H}^{1}$ norm, while well suited to measuring certain aspects of the deviation of the solution from the solitary 
wave, is not sensitive to dispersive phenomena. Dispersion must be taken into account, as in theorem 1 , if one is to deduce the asymptotic state of the system, for data beginning near a solitary wave.

The idea behind this result is now described. Suppose a solitary wave moving at speed $c>0$ to the right is perturbed slightly. One expects the perturbation to generate dispersive waves and possibly small additional solitary waves. It can be seen by examining the linear dispersion relation of $\mathrm{gKdV}$ that dispersive waves travel to the left. Also, small solitary waves of gKdV are known to move slower than large solitary waves. Thus the right moving solitary wave is expected to "outrun" the generated distortions. It is then reasonable to expect that in a suitably chosen frame, moving with the wave crest, the local energy of the perturbation will decay. The theorem states (i) in what sense the local energy of the perturbation can be measured, and (ii) that the decay in time is exponential. In contrast, one expects the global rate of decay to be algebraic; for example in the $\mathrm{L}^{\infty}$ norm the decay rate is expected to be $t^{-1 / 3}$.

The parametrization used for the first, and dominant term, in (1.3) can be motivated by observing that for fixed values of $c$ and $\gamma$, the leading term in (1.3) is an exact solitary wave solution of (1.1). If we perturb the solitary wave slightly, it is natural to expect the solitary wave to adjust, via slow and small variations of its available parameters, to a nearby solitary wave. Thus we allow the parameters $c$ and $\gamma$ to "modulate". The functions $c(t)$ and $\gamma(t)$, appearing in the decomposition (1.3), are sometimes referred to as collective coordinates. Equations for collective coordinates have been previously derived by various formalisms (see for example refs. [1618]). The equations governing these collective coordinates are sometimes called modulation equations. In formal perturbation theories, the coupling to the dispersion is neglected and the modulation equations are approximated by a coupled system of ordinary differential equations. The validity of this approximation on large but finite time intervals is considered in ref. [19].

To prove theorem 1 , the coupling of radiation to the bound state part must be studied. The main difficulty in proving an asymptotic stability result stems from the essential spectrum of linearized operator being the entire imaginary axis. This is a conse- quence of the Hamiltonian structure of $\mathrm{gKdV}$ and the $H^{1}$ orbital stability of the solitary wave [9]. The weighted norm introduced has the effect of shifting the essential spectrum off the imaginary axis into the left half plane. The neutral modes, arising from parameters associated with translation invariance and speed, remain eigenmodes with eigenvalue zero in the weighted space. These are "projected out" via modulation of the parameters $c$ and $\gamma$. Since the essential spectrum has been shifted to the left half plane, the reduced problem will be dissipative, provided the linearized operator in the unweighted space has no embedded eigenvalues in its essential spectrum. The exponential rate of decay of the perturbation in the weighted space is related to the distance of the resonance pole of the linear operator $\partial_{y} L_{c}$ (see below), which is in the left half plane and is nearest to the imaginary axis. (See sections 2, 3 and ref. [20].)

The technique of shifting the spectrum by introducing a weighted space has been used in dissipative problems to prove asymptotic stability. See, for example, the papers of Peletier [21] and Sattinger [22] on asymptotic orbital stability of traveling waves of nonlinear parabolic equations, see also ref [31]. In these papers, the essential spectrum of the linearized operator lies in the left half plane but touches the imaginary axis at the origin. Introducing a weighted space shifts the essential spectrum and uncovers the simple eigenvalue at zero associated with translating the wave. In these problems, it is possible to solve for, via the implicit function theorem, the "asymptotic phase shift", $\gamma_{\infty}$. In the present context, it appears convenient to allow the parameters $c$ and $\gamma$ to evolve, and let the system "discover" its asymptotic state.

\section{Decomposition into bound (solitary wave) and dispersive parts}

We seek solutions of the initial value problem of the form

$u(x, t)=u_{c(t)}(y(t))+v(y(t), t)$,

where

$y(t)=x-\int_{0}^{t} c(s) \mathrm{d} s+\gamma(t)$. 
Substitution into (1.1) gives the equation for the perturbation about the solitary wave:

$$
\begin{gathered}
\partial_{t} v=\partial_{y} L_{c(t)} v+\partial_{y}\left[h\left(u_{c(t)}, v\right) v\right]-\dot{\gamma}(t) \partial_{y} v \\
-\left(\dot{\gamma} \partial_{y}+\dot{c} \partial_{c}\right) u_{c(t)},
\end{gathered}
$$

where

$L_{c(t)} \equiv-\partial_{y}^{2}+c(t)-f^{\prime}\left(u_{c(t)}\right)$,

and

$h\left(u_{c(t)}, v\right) v \equiv f\left(u_{c(t)}+v\right)-f\left(u_{c(t)}\right)-f^{\prime}\left(u_{c(t)}\right) v$.

For the analysis, it is desirable to work with a reference linearized operator, $\partial_{y} L_{c 0}$, where the choice of $c_{0}$ is dictated by an initial decomposition. This is carried out along the lines of refs. [6-8]. The perturbation equation can then be rewritten as

$$
\begin{aligned}
& \partial_{t} v=\partial_{y} L_{c 0} v+\partial_{y}\left[c(t)-c_{0}-\dot{\gamma}(t)+f^{\prime}\left(u_{c 0}\right)\right. \\
& \left.-f^{\prime}\left(u_{c(t)}\right)+h\left(u_{c(t)}, v\right)\right] v-\left(\dot{\gamma} \partial_{y}+\dot{c} \partial_{c}\right) u_{c(t)} .
\end{aligned}
$$

The functions $\gamma(t)$ and $c(t)$ are to be determined so that the perturbation $v(\cdot, t)$ tends to zero as $t \rightarrow+\infty$ in a suitable norm.

Motivated by properties of the constant coefficient equation, $\partial_{t} z=\partial_{y}\left(-\partial_{y}^{2}+c\right) z=0$, we find that a natural space in which to study $v(\cdot, t)$ is an exponentially weighted space. Thus, we further transform the equation for $v$ by setting

$w \equiv \mathrm{e}^{\sigma y} v, \quad 0<\sigma<\sqrt{c_{0}}$.

Substitution into $(2.3)$ gives

$\partial_{t} w=A_{\sigma} w+\mathscr{F}$.

Here

$A_{\sigma}=\mathrm{e}^{\sigma y} \partial_{y} L_{c 0} \mathrm{e}^{-\sigma y}$,

and $\mathscr{F}$ is a source term.

We then introduce projection operators $P$ and $Q=I-P$, where $P$ denotes the spectral projection onto the generalized null space of $A_{\sigma}$,

$\mathrm{N}_{g}\left(A_{\sigma}\right)=\left\{\mathrm{e}^{\sigma y} \partial_{y} u_{c 0}, \mathrm{e}^{\sigma y} \partial_{c} u_{c 0}\right\}$,

and decompose the source term, $\mathscr{F}$ as

$\mathscr{F}=P \mathscr{F}+Q \mathscr{F}$.

We require that $P w(\cdot, t)=0$ for $t \geqslant 0$. The system (2.5) is then written in the form $\partial_{t} w=A_{\sigma} w+Q \mathscr{F}$,

$P \mathscr{F}=0$.

Eq. (2.6b) can be put in the form of a system of modulation equations for the parameters $c(t)$ and $\gamma(t)$ :

$\mathscr{A}(t)\left(\begin{array}{l}\dot{\gamma} \\ \dot{c}\end{array}\right)=\left(\begin{array}{l}R_{1} \\ R_{2}\end{array}\right)$.

Here

$\mathscr{A}(t)=I+\mathrm{O}\left(\left|c(t)-c_{0}\right|+\|v(\cdot, t)\|_{\mathrm{L}^{2}}\right)$

as $\left|c(t)-c_{0}\right|+\|v\|_{\mathrm{L}^{2}} \rightarrow 0 . R_{1}$ and $R_{2}$ are functions of time, which satisfy estimates of the form

$\left|R_{j}(t)\right| \leqslant C_{j}\left(\|v(\cdot, t)\|_{\mathrm{H}^{1}}\right)\|w(\cdot, t)\|_{\mathrm{H}^{1}}$.

Eq. (2.7) must be solved in tandem with (2.5), which we write in the equivalent integral form

$w(t)=\exp \left(A_{\sigma} t\right) w_{0}+\int_{0}^{t} \exp \left[A_{\sigma}(t-s)\right] Q \mathscr{F} \mathrm{d} s$.

Summarizing, we have that $w$ satisfies (2.8) and $\gamma(t)$ and $c(t)$ satisfy $(2.7)$ if and only if $u(x, t)$, given by $(2.1)$ is a solution of $(\mathrm{gKdV})$. Theorem 1 is proved via a priori estimates on the coupled system (2.7) and (2.8) using the tools outlined in the next section.

\section{Ingredients of the analysis}

The analysis is broken down into the following steps.

Step 1: linearized stability.

The propagator $\exp \left(\partial_{y} L_{c} t\right)$ is studied and we prove:

Proposition 2. $\mathrm{N}_{g}\left(L_{c} \partial_{y}\right)^{\perp}$ is an invariant subspace for the group $\exp \left(\partial_{y} L_{c} t\right)$. For any $g \in \mathrm{N}_{g}\left(L_{c} \partial_{y}\right)^{\perp}$,

$\sup _{t \in \mathbb{R}^{1}}\left\|\exp \left(\partial_{y} L_{c} t\right) g\right\|_{\mathrm{H}^{1}} \leqslant C\|g\|_{\mathrm{H}^{1}}$.

Step 2: absence of nonzero embedded eigenvalues. 
The essential spectrum of $\partial_{y} L_{c}$ is the entire imaginary axis and due to the translation invariance of (1.1), zero is a (generalized) eigenvalue of $\partial_{y} L_{c}$ [9]. In subsequent steps we require the following important property of the operator $\partial_{y} L_{c}$ :

(E) Zero is the only embedded eigenvalue in the essential spectrum of $\partial_{y} L_{c}$.

We have proved:

Proposition 3. Consider ( $\mathrm{gKdV}$ ) with nonlinearity $f(u)=u^{p+1} /(p+1), p \geqslant 1$.

(a) For $p=1$ (E) holds.

(b) The set $\{p \in(1, \infty):(E)$ does not hold $\}$ is discrete and has no accumulation point. In particular, in the range where the solitary wave, $u_{c}$, is $\mathrm{H}^{1}$ Lyapunov stable, $1 \leqslant p<4$, (E) fails to hold at most at a finite number of values of $p$.

For part (a) we explicitly construct Evans' function, $D(\lambda)$ [9,23-28]. In ref. [9], we proved that the zeroes of $D(\lambda)$ in the closed right half plane are in one to one correspondence with the the eigenvalues of $\partial_{y} L_{c}$. The construction is carried out using formulas known for the linearized problem of $\mathrm{KdV}, p=1$ (see ref. [29] ). To prove part (b), we use part (a), the analyticity of $D(\lambda ; p)$, and the fact that $\partial_{y} L_{c}$ can have no more than one eigenvalue in the open right half plane for any value of $p$. This latter assertion follows from a general bound, giving constraints on the spectrum of operators of the form $J L$, where $J$ is skew symmetric and $L$ is self-adjoint with $k$ negative eigenvalues [9]. Numerical evidence suggests that (E) holds for all $p \geqslant 1$.

Step 3: smoothing and decay estimates.

Our aim is to show that the weighted perturbation $w=\mathrm{e}^{\sigma v} v$ behaves dissipatively. This is related to work of Sattinger [22] and Kato [30]. The weighted perturbation $w=\mathrm{e}^{\sigma y} v$ is constructed to lie in $\operatorname{Range}(Q)$ (see $(2.6 \mathrm{~b})$ ). The parameter $\sigma$ is chosen so that $0<\sigma<\sqrt{c_{0}}$. A contour $\Gamma$, is chosen in the left half plane so that $\sigma\left(A_{\sigma}\right)-\{0\}$ lies to the left of $\Gamma$ and so that we can represent the semigroup $\exp \left(A_{\sigma} t\right)$ as a Cauchy integral:

$\exp \left(A_{\sigma} t\right)=\frac{1}{2 \pi \mathrm{i}} \int_{\Gamma} \exp (\zeta t)\left(\zeta I-A_{\sigma}\right)^{-1} \mathrm{~d} \zeta$.

\section{The key estimate is}

Proposition 4. Let $p<4$ and $0<\sigma<\sqrt{c}$. Then, there is a positive number, $\delta=\delta\left(\sigma, c_{0}, p\right)$, such that

$\left\|\exp \left(A_{\sigma} t\right) Q g\right\|_{\mathrm{H}^{1}} \leqslant C t^{-1 / 2} \exp (-\delta t)\|g\|_{\mathrm{L}^{2}}$.

The exponential decay rate, $\delta\left(\sigma, c_{0}, p\right)$, depends on the singularity of the resolvent of $A_{\sigma}$ in the left half plane, which is closest to the imaginary axis. It can be shown, subject to the condition that $(E)$ holds for $p=4$, that for $0<4-p<\epsilon$, with $\epsilon$ sufficiently small, this singularity is a resonance pole of the operator $\partial_{y} L_{c}$, which lies on the real axis. (Numerical simulations strongly suggest that (E) holds for $p=4$.) As $p \rightarrow 4^{-}$, this resonance pole approaches zero [9]. Therefore, the exponential rate, with which the $\mathrm{H}_{o}^{1}$ norm of the fluctuation about the solitary wave decays, decreases. At $p=4$ stability breaks down $[9,11]$.

Step 4: a priori estimates and asymptotic behavior.

Theorem 1 is proved via a priori estimates on the coupled system (2.7) and (2.8) using the estimates of steps $1-3$. The estimates obtained are

$$
\begin{aligned}
& \sup _{0 \leqslant \tau<T}\left[\langle\tau\rangle^{1 / 2} \exp (\delta \tau)\|w(\tau)\|_{\mathrm{H}^{1}}\right] \leqslant C_{1}, \\
& \sup _{0 \leqslant \tau<T}\left[\langle\tau\rangle^{1 / 2} \exp (\delta \tau)|\dot{c}(\tau)|\right] \leqslant C_{2}, \\
& \sup _{0 \leqslant \tau<T}\left[\langle\tau\rangle^{1 / 2} \exp (\delta \tau)|\dot{\gamma}(\tau)|\right] \leqslant C_{3}, \\
& \sup _{0 \leqslant \tau<T}\|v(\cdot, \tau)\|_{\mathrm{H}^{\prime}} \leqslant C_{4},
\end{aligned}
$$

where $\langle\tau\rangle=\left(1+\tau^{2}\right)^{1 / 2}$.

The constants $C_{1}, C_{2}, C_{3}$ and $C_{4}$ are independent of $T$ but depend on the $\mathrm{H}^{1}$ and weighted $\mathrm{H}^{1}$ norms of the initial data, which are assumed to be sufficiently small, so that the set of a priori estimates to close. A Lyapunov functional, in the sense of the $\mathrm{H}^{1}$ stability analysis, is used together with estimates on $c(t), \gamma(t)$ and $w(\cdot, t)$ to control $\|v(\cdot, t)\|_{\mathrm{H}^{1}}$.

\section{References}

[1] M.J. Ablowitz and H. Segur, Solitons and the inverse scattering transform (SIAM, Philadelphia, 1981). 
[2] C.S. Gardner, J.M. Greene, M.D. Kruskal and R.M. Miura, Phys. Rev. Lett. 19 (1967) 1095.

[3] T.B. Benjamin, Proc. R. Soc. A 328 (1972) 153.

[4] D.J. Korteweg and de Vries, Philos. Mag. 39 (1895) 422.

[5] E.A. Coddington and N. Levinson, Theory of ordinary differential equations (McGraw-Hill, New York, 1955).

[6] A. Soffer and M.I. Weinstein, Multichannel nonlinear scattering theory for nonintegrable equations, Integrable Systems and Applications, in: Springer lecture notes in physics, Vol. 342, eds. M. Balabane, P. Lochak and C. Sulem.

[7] A. Soffer and M.I. Weinstein, Commun. Math. Phys. 133 (1990) 119.

[8]A. Soffer and M.I. Weinstein, Multichannel nonlinear scattering theory for nonintegrable equations II: The case of anisotropic potentials and data, J. Diff. Eq., in press.

[9] R.L. Pego and M.I. Weinstein, A class of eigenvalue problems with applications to solitary wave instabilities, IMAMinneapolis preprint no. 863 (August 1991).

[10] J.L. Bona, Proc. R. Soc. A 344 (1975) 363.

[11] J.L. Bona, P.E. Souganides and W.A. Strauss, Proc. R. Soc. A 411 (1987) 395.

[12] W.E. Laedke and K.H. Spatschek, J. Plasma Phys. 32 (1984) 263.

[13] M.I. Weinstein, On the solitary wave of the generalized Korteweg-de Vries equation, in: Proc. Santa Fe Conf. on Nonlinear PDE, July 1984, Lectures in Appl. Math., Vol. 23 (1986).

[14] M.I. Weinstein, Commun. Pure Appl. Math. 39 (1986) 51.

[15] M.I. Weinstein, Commun. P.D.E. 12 (1987) 1133.
[16] Y. Kodama and M.J. Ablowitz, Stud. Appl. Math. 64 (1981) 225.

[17] J.P. Keener and D.W. McLaughlin, Phys. Rev. A 16 (1977) 777.

[18] A.C. Newell, in: nonlinear evolution equations solvable by the inverse spectral transform, ed. F. Calogero (Pitman, London, 1978) pp. 127-179.

[19] M.I. Weinstein, SIAM J. Math. Anal. 16 (1985) 472.

[20] R.L. Pego and M.I. Weinstein, Asymptotic stability of solitary waves, in preparation.

[21] L.A. Peletier, On the stability of waves of nonlinear parabolic systems, in: Proc. IUTAM Conference (Springer, Berlin, 1971).

[22] D.H. Sattinger, Adv. Math. 22 (1976) 312.

[23] J. Alexander, R. Gardener and C. Jones, Z. Reine Angew. Math. 410 (1990) 167.

[24] J.W. Evans, Indiana Univ. Math. J. 21 (1972) 877.

[25] J.W. Evans, Indiana Univ. Math. J 22 (1972) 75.

[26] J.W. Evans, Indiana Univ. Math. J 22 (1972) 577.

[27] J.W. Evans, Indiana Univ. Math. J. 24 (1975) 1169.

[28] C.K.R.T. Jones, Trans. Am. Math. Soc. 286 (1984) 431.

[29] A. Jeffrey and Kakutani, Indiana Univ. Math. J. 20 (1971) 463.

[30] T. Kato, On the Cauchy problem for the (generalized) Korteweg-de Vries equation, in: Studies in applied mathematics supplementary series, Vol. 8 (Academic Press, New York, 1983).

[31] D. Henry, Geometric theory of semilinear parabolic equations (Springer, Berlin, 1981). 